\section{OPEN ACCESS}

Edited by:

Maria Isabel Colombo,

Universidad Nacional de

Cuyo, Argentina

Reviewed by:

Laurent Gorvel,

INSERM U1068 Centre de Recherche en Cancérologie de Marseille, France

Patricia Silvia Romano,

National Scientific and Technical

Research Council, Argentina

*Correspondence:

Carolina Verónica Poncini cvponcini@gmail.com

Specialty section:

This article was submitted to Microbes and Innate Immunity,

a section of the journal

Frontiers in Cellular and Infection Microbiology

Received: 04 September 2019 Accepted: 14 January 2020

Published: 27 February 2020

Citation:

Gutierrez BC, Lammel E, Ramirez MI, González-Cappa SM and Poncini CV

(2020) Modulatory Effect of

Trypanosoma cruzi Infective Stages in

Different Dendritic Cell Populations

in vitro.

Front. Cell. Infect. Microbiol. 10:20.

doi: 10.3389/fcimb.2020.00020

\title{
Modulatory Effect of Trypanosoma cruzi Infective Stages in Different Dendritic Cell Populations in vitro
}

\section{Brenda Celeste Gutierrez ${ }^{1}$, Estela Lammel ${ }^{1}$, Marcel Ivan Ramirez ${ }^{2,3}$, Stella Maris González-Cappa ${ }^{1,4}$ and Carolina Verónica Poncini ${ }^{1,4 *}$}

' Laboratório de Inmunología Celular e Inmunopatología de Infecciones, Instituto de Investigaciones en Microbiología y Parasitología Médica (MPaM) UBA-CONICET, Buenos Aires, Argentina, ${ }^{2}$ Instituto Oswaldo Cruz, FIOCRUZ, Rio de Janeiro, Brazil, ${ }^{3}$ Departamento de Bioquímica, Universidade Federal Do Paraná, Curitiba, Brazil, ${ }^{4}$ Departamento de Microbiología, Parasitología e Inmunología, Facultad de Medicina, Universidad de Buenos Aires, Buenos Aires, Argentina

Trypanosoma cruzi is a protozoan parasite that infects at least 7 million persons in the world (OMS, 2019). In endemic areas, infection normally occurs by vectorial transmission; however, outside, it normally happens by blood and includes congenital transmission. The persistence of $T$. cruzi during infection suggests the presence of immune evasion mechanisms and the modulation of the anti-parasite response to a profile incapable of eradicating the parasite. Dendritic cells (DCs) are a heterogeneous population of antigen-presenting cells (APCs) that patrol tissues with a key role in mediating the interface between the innate and adaptive immune response. Previous results from our lab and other groups have demonstrated that $T$. cruzi modulates the functional properties of DCs, in vitro and in vivo. During vectorial transmission, metacyclic $(\mathrm{m})$ trypomastigotes (Tps) eliminated along with the insect feces reach the mucous membranes or injured skin. When transmission occurs by the hematic route, the parasite stage involved in the infection is the circulating or blood (b) Tp. Here, we studied in vitro the effect of both infective $\mathrm{mTp}$ and bTp in two different populations of DCs, bone marrow-derived DCs (BMDCs) and XS106, a cell line derived from epidermal DCs. Results demonstrated that the interaction of both Tps imparts a different effect in the functionality of these two populations of DCs, suggesting that the stage of T. cruzi and DC maturation status could define the immune response from the beginning of the ingress of the parasite, conditioning the course of the infection.

Keywords: Trypanosoma cruzi, dendritic cells, cell activation, cytokines, T cells

\section{INTRODUCTION}

Trypanosoma cruzi, the etiological agent of Chagas disease, is a protozoan parasite that affects 7 million people in the world (OMS, 2019).

The parasite life cycle includes at least three particular morphological stages. In the insect vector, there is the presence of multiplicative epimastigotes and infective metacyclic trypomastigotes (mTp), released with feces. In the mammal host, two stages are present, the intracellular multiplicative amastigotes and bloodstream trypomastigotes (bTp), involved in the parasite dissemination to tissues. While vectorial and oral transmission involves mTp entry via injured skin or mucous membranes, parasite dissemination in the mammalian host occurs by bTp, also 
responsible for congenital and sanguineous transmission even in non-endemic regions (de Souza et al., 2014; OMS, 2019).

A limited number of studies compare the immune response against different $T$. cruzi infective stages.

In a comparative study between mTp and bTp, Dias et al. (2013) have demonstrated that the infection by intraperitoneal or oral inoculation of bTp is more virulent than with mTp. Specifically, animals infected with bTp presented higher parasitemia and mortality in comparison to infection with mTp (Dias et al., 2013).

Dendritic cells (DCs) are professional antigen-presenting cells (APCs) with a central role in the development of immunity against infections. In steady state, DCs patrol tissues in order to maintain immune homeostasis. Under certain conditions such as inflammation or infection, these cells can be activated after recognizing danger signals. This process includes uptake and processing of antigens for presentation in addition to DC migration to draining lymph nodes (LNs). DCs are responsible for $\mathrm{T}$-cell priming, the activation of secondary immune responses, or the induction of tolerogenic programs (Merad et al., 2013).

Previous studies from our group demonstrated that bTp from the virulent strain RA (Gonzalez Cappa et al., 1981) negatively regulates bone marrow-derived DC (BMDC) activation in vitro. The parasite promotes the production of anti-inflammatory cytokines and diminishes the T-cell stimulatory capacity of DCs (Poncini et al., 2008). In addition, our results demonstrate that both live bTp and heat-killed $\mathrm{Tp}$, but not fixed $\mathrm{Tp}$, condition DCs to a tolerogenic profile, suggesting that TpDC surface interaction and not infection triggers the DCs polarization. In fact, we confirmed that early in time, TpDC interaction induces ERK phosphorylation and IL-10 upregulation via a TLR-4-dependent pathway (Poncini et al., 2010).

In a previous report, da Costa et al. (2014) have shown that $\mathrm{Tp}$ from strains with variable virulence differentially modulates DC activation in vitro. In vivo, it was demonstrated that during infection, splenic DCs and APCs display low expression of MHCII and/or costimulatory molecules and impaired function (Alba Soto et al., 2003; Planelles et al., 2003), and more recently, that DCs with tolerogenic properties promote Treg cell induction and parasite persistence (Poncini et al., 2015). The fact that the parasite successfully infects the mammalian host via different portals of entry and by different infective stages highlights the importance the model of infection acquires during experimentation. As a result, a detailed characterization of the interaction of the parasite with cells involved in the first line of defense during primoinfection was needed (Poncini and González-Cappa, 2017).

This work comparatively describes the effect of two different infective stages of a virulent strain of T. cruzi (RA) in DCs from different origins. By studying the interaction of bTp and mTp with BMDCs and XS106, a cell line derived from epidemic DCs (Mohan et al., 2005), we found an interesting approach to understand and hypothesize about the different responses that may be occurring at the parasite entry site.

\section{MATERIALS AND METHODS}

\section{Animals}

$\mathrm{C} 3 \mathrm{H} / \mathrm{HeN}, \mathrm{C} 57 \mathrm{BL} / 6$, and $\mathrm{CF} 1$ mice were maintained in the animal facilities of IMPaM UBA-CONICET, Facultad de Medicina, Universidad de Buenos Aires, and bred under a sanitary barrier in specific-pathogen-free conditions (Poncini et al., 2015).

All experiments were performed according to protocol CD $\mathrm{N}^{\circ}$ 04/2015 approved by the University of Buenos Aires's Institutional Committee for the Care and Use of Laboratory Animals (CICUAL) in accordance with the Council for International Organizations of Medical Sciences (CIOMS) and International Council for Laboratory Animal Science (ICLAS) and the international ethical guidelines for biomedical research involving animals.

\section{Parasites}

RA T. cruzi bTps were maintained in CF1 mice and obtained from whole blood at the peak of parasitemia (7 days postinfection) by differential centrifugation or by density gradient, using Histopaque-1083 (Sigma-Aldrich) as previously reported (Poncini et al., 2008). Parasites were obtained from the supernatant by centrifugation $\left(10,000 \mathrm{~g}, 30 \mathrm{~min}\right.$, and $\left.20^{\circ} \mathrm{C}\right)$ and resuspended in fresh Iscove's modified Dulbecco's medium (IMDM, Sigma-Aldrich). Epimastigotes were cultured in vitro in liver infusion tryptose (LIT) medium at $27^{\circ} \mathrm{C}$ to the exponential phase of growth and centrifuged at $3,000 \mathrm{~g}$ for $15 \mathrm{~min}$ at $10^{\circ} \mathrm{C}$. The flasks had a liquid depth not exceeding $10 \mathrm{~mm}$ and were incubated without agitation for different amounts of time according to the experimental schedule (Isola et al., 1986). mTps were obtained as described by Cestari et al. (2012) with some adjustments. Briefly, after in vitro culture of $10 \times$ $10^{7}$ epimastigotes in $10 \%$ fetal bovine serum (FBS) LIT plus Grace's insect medium (MERC) and incubation at $27^{\circ} \mathrm{C}$ in tightly closed culture flasks, parasites were purified in a DEAE column equilibrated with PBS-glucose (20\%) at pH 8.2. Purity was analyzed by microscopic examination.

\section{BMDCs and XS106 Cell Line Cultures}

Bone marrow from $\mathrm{C} 3 \mathrm{H} / \mathrm{HeN}$ mice was cultured for 7 days as previously described (Poncini et al., 2008). Briefly, bone marrow was flushed from femurs and tibias by syringe and 25-gauge needles with IMDM supplemented with 10\% FBS, $100 \mathrm{U} / \mathrm{ml}$ penicillin, $100 \mathrm{mg} / \mathrm{ml}$ streptomycin, and $50 \mu \mathrm{M} \mathrm{2-}$ mercaptoethanol (referred to below as 10-IMDM). The tissue was mechanically disaggregated, and DCs were obtained by culturing BM cells, supplemented with $20 \%$ supernatant from a GM-CSF-expressing cell line (J558 GM-CSF) at $37^{\circ} \mathrm{C}$ and $5 \%$ CO2. Then, at days 2 and 5, fresh medium was added to the cultures. At day 7 , cells displayed a myeloid phenotype (>95\% CD11b) and were highly enriched in DCs $(>70$ $\% \mathrm{CD} 11 \mathrm{c})$.

XS106, a long-term DC line established from newborn epidermis of A/J mice, was kindly provided by Dr. Takashima (University of Toledo, MTA M2014-25). They express a mature phenotype as described by Mohan et al. (2005). 
Then, cells were harvested, washed, and plated $\left(1 \times 10^{6}\right.$ cells/ml) in 24-well plates (Nunc, NY, USA) and cultured in conditioned medium (Mohan et al., 2005) with or without $10 \mu \mathrm{g} / \mathrm{ml}$ LPS (Escherichia coli O26:B6, Sigma-Aldrich), and/or $\mathrm{b} / \mathrm{mTp}$ (cell-to-parasite ratio $1: 1,1: 4$, or $1: 10$, depending on the experiment) overnight (ON).

\section{BMDCs and XS106 Cell Culture, Stimulation, and Infection}

As described above, cells were cultured in 24-well plates (Nunc, NY, USA) in medium with bTp or mTp (cell-to-parasite ratio $1: 1,1: 4$, or $1: 10$, depending on the experiment) $\mathrm{ON}$ at $37^{\circ} \mathrm{C}$ and $5 \% \mathrm{CO}_{2}$. Then, cells were washed to eliminate parasites in suspension and cultured in fresh medium for 4 or 7 more days. Then, samples were processed by cytospin and cell imprints stained with Giemsa. For immunofluorescence microscopy, cells were washed and stained with anti-mouse CD11c PE-conjugated $\mathrm{mAb}$ (BD PharMingen), attached to positively charged glass slides (Fisherbrand, Pittsburgh, PA), fixed in methanol, and stained with anti-parasite rabbit serum or appropriate controls and FITC-conjugated secondary Ab (Sigma-Aldrich).

The percentages of infected cells at the different cell-Tp ratios were defined after microscopic examination $(400 \times$ or 1,000 $\times$ magnification) by quantifying the relative number of cells with amastigotes in 15 random fields counted per treatment per sample.

\section{Mixed Lymphocyte Reaction}

To characterize APC capacity to induce lymphoproliferation, BMDCs or XS106 cells were cocultured with lymphocytes to test alloresponse as previously reported (Poncini et al., 2008). Briefly, DCs were cultured with different stimuli (medium, LPS, bTp/mTp) for $24 \mathrm{~h}$, harvested, washed, irradiated $(30 \mathrm{~Gy})$, and plated with single-cell suspension enriched in $\mathrm{T}$ cells prepared from LNs of 8 -week-old male C57BL/6 mice after $\mathrm{CD}^{+}$T-cell purification by positive selection (MiniMACS separation; Miltenyi Biotec). Briefly, $\mathrm{T}$ cells $\left(\mathrm{CD}^{+}\right)$were purified from $\mathrm{LN}$ by using anti-mouse biotin-conjugated CD3 mAb (145-2C11; BD Biosciences) and streptavidin-conjugated microbeads for magnetic positive selection (MiniMACS). Purity was checked by flow cytometry and found to be around $90 \%$ of $\mathrm{CD}^{+}$cells, as previously reported (Poncini et al., 2015). Cells were plated at a 1:10 APC-LN cell ratio, using $1 \times 10^{5} \mathrm{LN}$ cells/well, and cultured at $37^{\circ} \mathrm{C}$ and 5\% CO2 in 10-RPMI 1640 medium (Gibco, NY, USA) supplemented with $2 \mathrm{mM}$ L-glutamine, $100 \mathrm{U} / \mathrm{ml}$ penicillin, $100 \mathrm{mg} / \mathrm{ml}$ streptomycin, and $50 \mu \mathrm{M}$ 2-mercaptoethanol. Mixed lymphocyte reaction (MLR) assay was performed in 96-well microplates (Nunc) and cultured for 3 days. Proliferation was quantified by Ki-67 (REA183, Miltenyi Biotec) detection by flow cytometry according to the manufacturer's protocol.

\section{Flow Cytometry}

Cells $\left(1 \times 10^{6}\right)$ were washed in ice-cold PBS supplemented with $1 \%$ bovine serum albumin and $0.1 \% \mathrm{NaN}_{3}$, and incubated for $30 \mathrm{~min}$ at $4^{\circ} \mathrm{C}$ with a previously optimized amount of one or more of the following anti-mouse mAbs conjugated to different fluorophores: CD11b-PE (M1/70), CD11c-PE (HL3), I-Ak-FITC (11-5.2), CD40-Biot (3/23), CD80-Biot (16-10A1), and CD86Biot (GL-1). Finally, cells were fixed with $1 \%$ paraformaldehyde. All $\mathrm{mAbs}$ and second reagents were purchased from PharMingen or Miltenyi Biotec. Samples were acquired on FACSCalibur (Becton Dickinson), and data were analyzed with FlowJo 7.6.2 software.

\section{Enzyme Linked Immuno Assay}

Cell culture supernatants were collected and stored at $-80^{\circ} \mathrm{C}$ until used. Mouse IL-10 and TNF- $\alpha$ were detected by enzyme linked immune assay (ELISA) (R\&D Systems) according to the manufacturer's protocol.

\section{Statistical Analysis}

Two groups were compared with unpaired Student's $t$-test. ANOVA and Bonferroni's or Dunnett's multiple comparison tests were performed in order to analyze statistical significance. All analyses were carried out with GraphPad Prism 4 software for Windows. A $p<0.05$ value was assumed as significant.

\section{RESULTS}

\section{XS106 Cell Line Phenotypic Characterization}

As previously reported by Mohan et al. (2005), the XS106 cell line presented surface expression of CD11b and CD11c integrin in accordance with DCs of myeloid origin. In contrast to BMDCs that, in steady state, display low expression of activation markers (Poncini et al., 2008), XS106 cells showed high CD40, CD86, and MHCII surface expression. We also detected the macrophage related marker F4/80 and low expression of the monocytic markers Ly6C and CD207 in XS106, resembling Langerhans cells or migratory DCs in the skin (Figure 1A).

Previous studies have demonstrated that at certain conditions, bTp alone does not affect the activation of BMDCs, measured as MHCII, CD40, CD80, and CD86 surface expression (Poncini et al., 2008). Analyzing here the stimulatory effect of LPS, bTp, or mTp in the XS106 cell line, we found that while these cells displayed a higher basal activation status than BMDCs, neither LPS nor the parasite alone (from 1:1 to $1: 10$ cell-to-Tp relation, data not shown) enhanced the expression of CD40, CD86, or MHCII. However, LPS plus bTp starting at a 1:4 cell-to-bTp ratio increased the expression of MHCII and the costimulatory molecules CD40 and CD86 (Figure 1B).

The parasite itself, bTp or mTp alone, did not modify the activation status of XS106 in culture (Figure 1C). These results were also observed at 1:10 XS106 cell-Tp relation (data not shown). All data suggest that while bTp can polarize the activation of steady-state BMDCs (Poncini et al., 2008), the parasite could not modify the status of mature DCs such as XS106. 


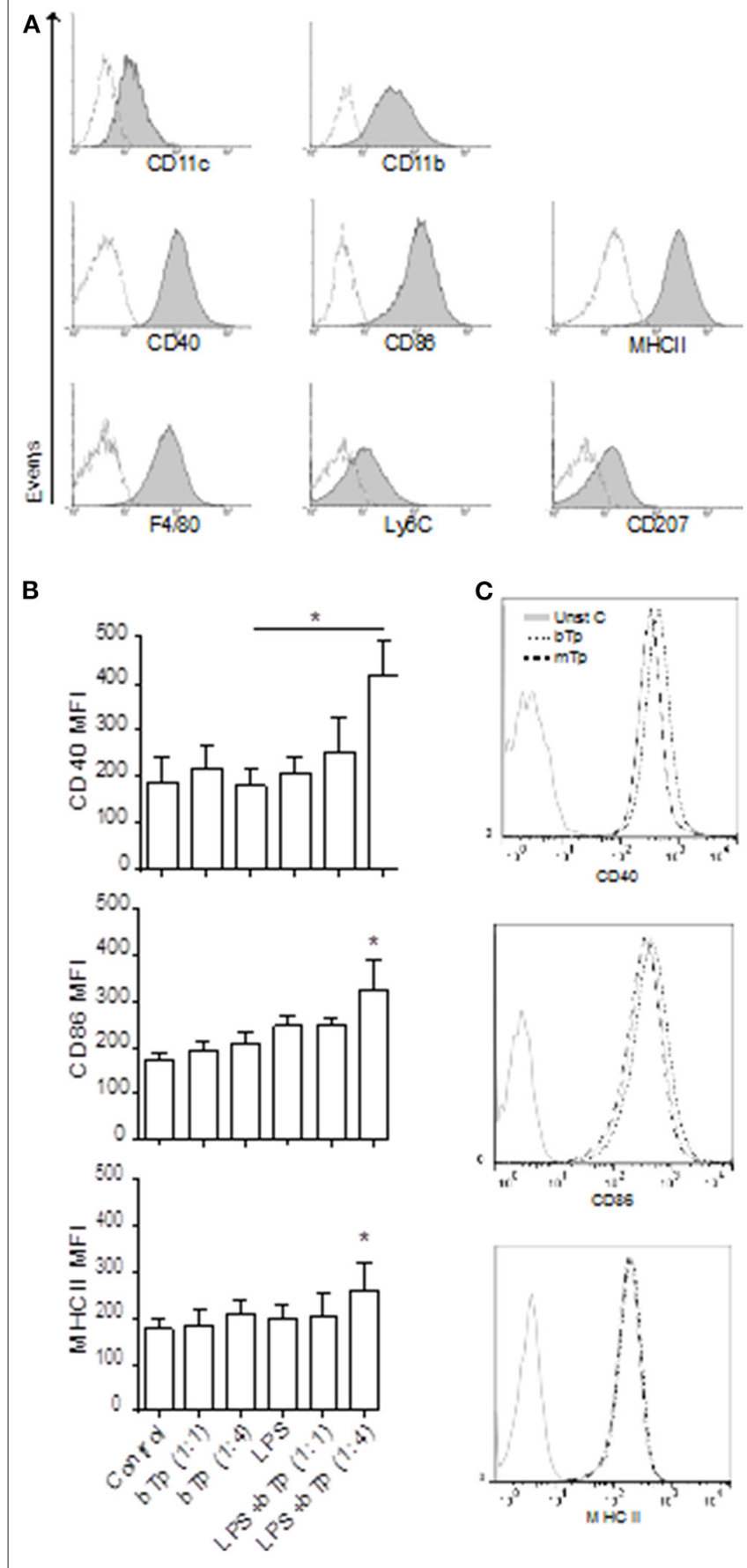

FIGURE 1 | Surface markers in XS106 cell line and comparative expression of activation markers in XS106 stimulated with bTp \pm LPS or mTp. (A) XS106 were cultured at $1 \times 10^{6} /$ well, stained with anti-mouse CD11c, CD11b, CD40, CD86, MHCII, F4/80, Ly6C, CD207 monoclonal Abs and analyzed by flow cytometry. One independent experiment of five is represented by histograms. Dotted line represents negative control for the staining. (B,C) $5 \times 10^{5}$ cells XS106 were incubated for $24 \mathrm{~h}$ with medium (control), bTp with or without LPS (B), and bTp vs. mTp (C). CD40, CD86, and MHCll were analyzed by flow cytometry. Bar graphic represents MFI (mean fluorescence intensity), mean \pm SEM $(n=5),{ }^{*} p<0$. 05. One-way ANOVA and Bonferroni's post-hoc correction.

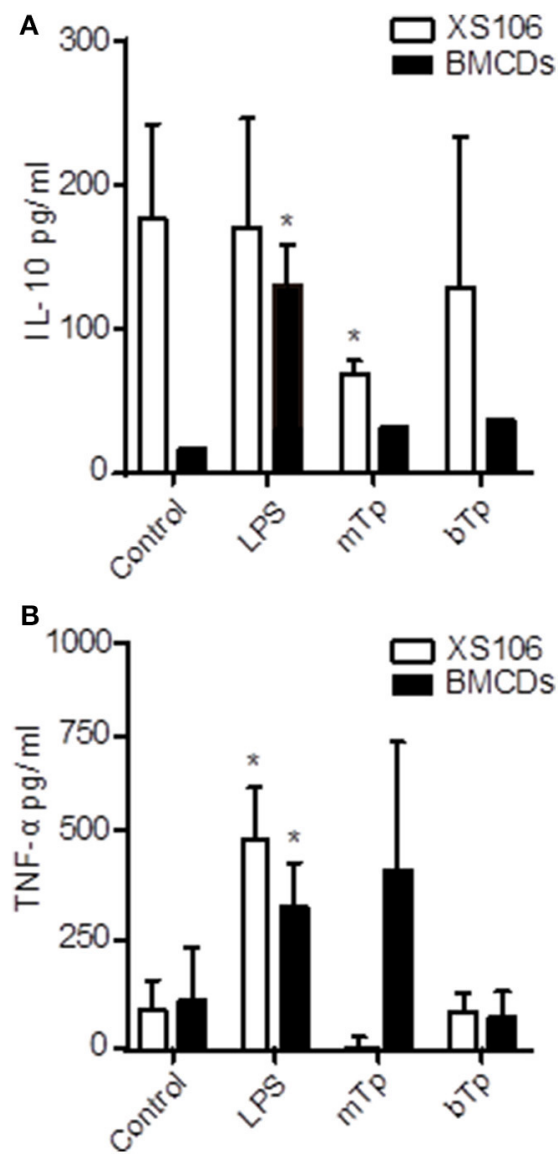

FIGURE 2 | Different IL-10 and TNF- $\alpha$ production patterns in XS106 cell line and BMDCs. $5 \times 10^{5}$ XS106 cells or BMDCs were incubated for $24 \mathrm{~h}$ in 10-IMDM (control), LPS, bTp or mTp at a parasite to cell ratio of 4. IL-10 (A) and TNF- $\alpha$ (B) were measured in culture supernatants by ELISA. ND, not detected. The results are presented as the mean \pm SEM of seven independent experiments, ${ }^{*} p<0.05$ (compared to the respective control). One-way ANOVA and Dunnett's post Test.

\section{Comparative Production of TNF and IL-10 in BMDC and XS106 Stimulated With $T$.} cruzi Infective Forms

Considering the basal activation of XS106, next we analyzed the production of TNF- $\alpha$ and IL-10, two cytokines with antagonistic properties in culture supernatants. XS106 and BMDCs were stimulated in vitro as described in Materials and Methods, and then, TNF- $\alpha$ and IL-10 were measured in culture supernatants as previously described by ELISA (Poncini et al., 2008).

XS106 showed a basal production of IL-10, which was downregulated in the presence of the parasite. The decline in the IL-10 production in XS106 was significant for $\mathrm{mTp}$. As expected for BMDCs, only LPS increased IL-10 secretion (Figure 2A). TNF- $\alpha$ was upregulated by LPS but not by $\mathrm{Tp}$ in XS106. In contrast, in BMDCs, both LPS and $\mathrm{mTp}$ enlarged the production of this cytokine (Figure 2B), but 
it was significant only for LPS, results consistent with cellular activation.

\section{T. cruzi Infectivity in BMDC vs. XS106 Cell Line}

To characterize the invasion and multiplication of the infective stages of T. cruzi bTp and mTp in XS106 cells and BMDCs, the parasites were cocultured with cells as described in Materials and Methods. Infection was determined and quantified by optic or fluorescence microscopy. Both XS106 cells and BMDCs presented intracellular amastigotes, confirming successful infection by bTp at 8 days post-infection (Figure 3A). However, XS106 cells were two times more infected with bTp than with $\mathrm{mTp}$ at the highest ratio of parasites (Figures 3B,C), and in comparison with BMDCs (Figure 3C). In BMDCs, no significant difference was detected in the percentage of infection between bTp and mTp (Figure 3C). These results suggest that at a high ratio of parasite to cell, bTp found a more conducive niche for multiplication in the XS106 cell line than in BMDCs.

\section{Effect of T. cruzi Infective Forms on BMDC and XS106 Capacity to Induce Lymphoproliferation}

Previous studies demonstrated that acute infection affects the maturation state of APCs and impairs the T-cell stimulatory capacity of splenic DCs (Alba Soto et al., 2003). In addition, in vitro and in vivo, we have demonstrated the ability of T. cruzi to modulate the differentiation of tolerogenic DCs (Poncini et al., 2008, 2015). In vitro, bTps fail to activate BMDCs, prevent the full activation by LPS, and induce high IL-10 secretion and a poor alloresponse (Poncini et al., 2008). Here we comparatively studied the antigen-presenting capacity of both XS106 cells and BMDCs after the stimulation with bTp, mTp, and LPS (positive control) in a model of alloresponse in an MLR.

As expected, LPS stimulated the antigen-presenting capacity of BMDCs. Despite not being significant, BMDCs cocultured with bTp presented less capacity to induce lymphoproliferation than mTp-treated BMDCs, which enhanced BMDCs' lymphoproliferative properties (Figures 4A,B). Interestingly, XS106 cells treated with bTp or mTp displayed enhanced capacity to induce T-cell proliferation compared with controls (Figures 4A,B). In addition, bTp-treated XS106 showed better antigen presentation than BMDCs (Figure 4B).

All these results demonstrate that the interaction of different T. cruzi infective stages with diverse APCs, but not the cell invasion and the parasite multiplication, could condition the antigen-presenting capacity of different DCs subsets early.

\section{DISCUSSION}

The aim of this work was to characterize the effect of two infective stages of T. cruzi on APCs, particularly DCs from a different origin. Previously, our group has demonstrated that bTps promote tolerogenic BMDCs in vitro (Poncini et al., 2008). Here, we comparatively studied the interaction of $\mathrm{mTp}$, the representative stage involved in vectorial transmission via the

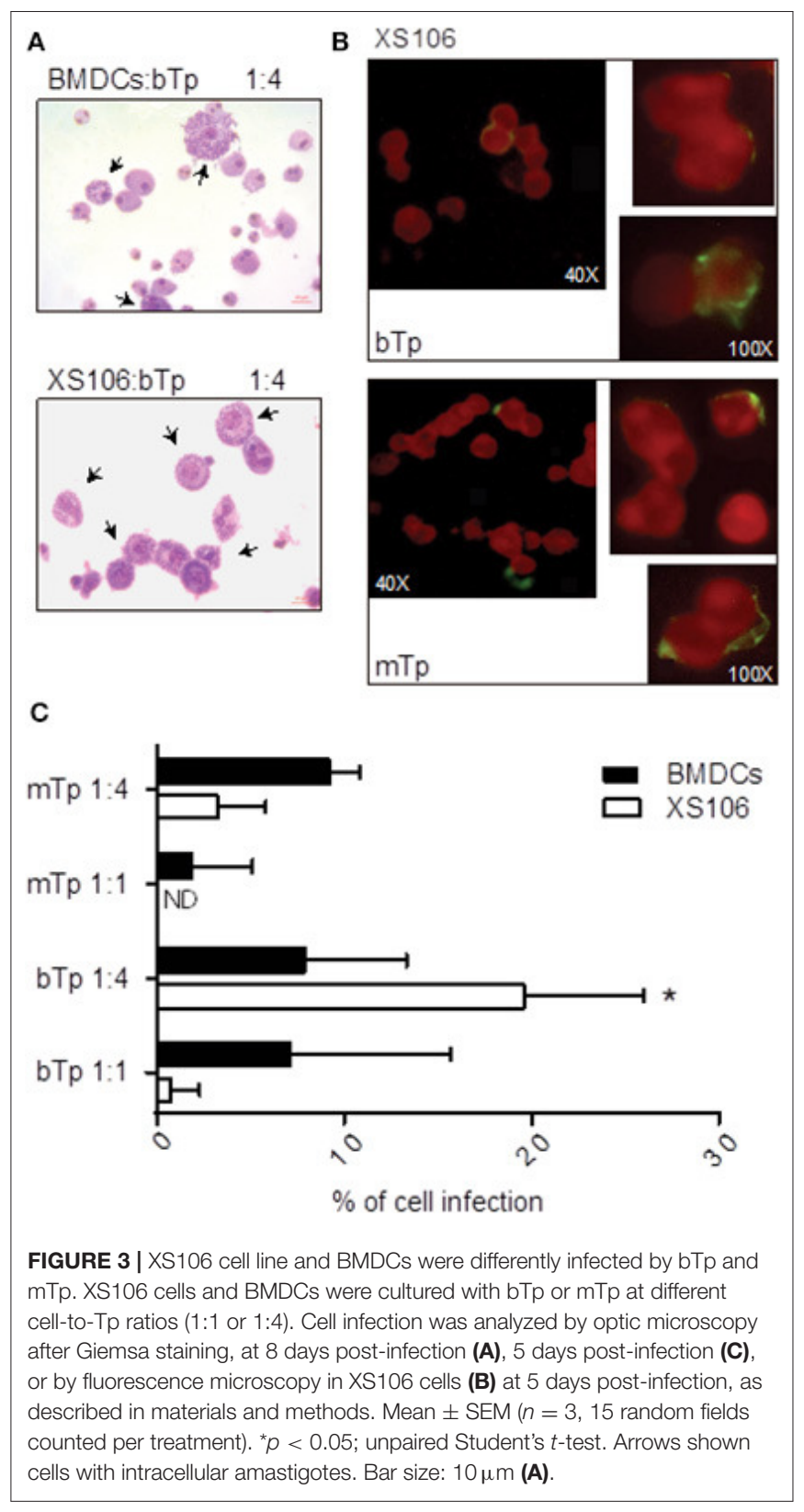

skin or mucous membranes (and gastric epithelium), and bTp responsible for transfusion and congenital transmission with two populations of DCs. The interaction was analyzed in vitro by using two different subsets of APCs that could interact with each of the stages described at different biological barriers: on one hand, XS106, a cell line obtained from skin epithelial DCs that resembles LCs (Mohan et al., 2005), and on the other, BMDCs, related to monocyte-derived (Mo)-DCs (Lutz et al., 2016).

The interest in XS106 cell-parasite interaction arises since they are similar to some of the APCs present in the skin. In fact, they presented intermediate expression of CD207 and F4/80 markers, commonly displayed in LCs, several migrating DCs, and some cells from the macrophage linage (Otsuka et al., 2018). In contrast to BMDCs, XS106 displayed 


\section{A}

\section{MLR \\ BMDCs}
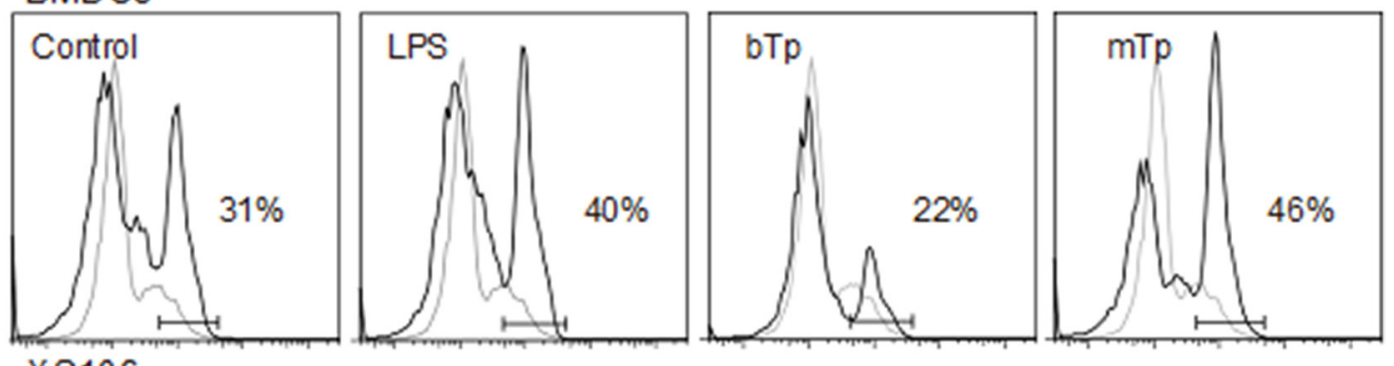

XS106
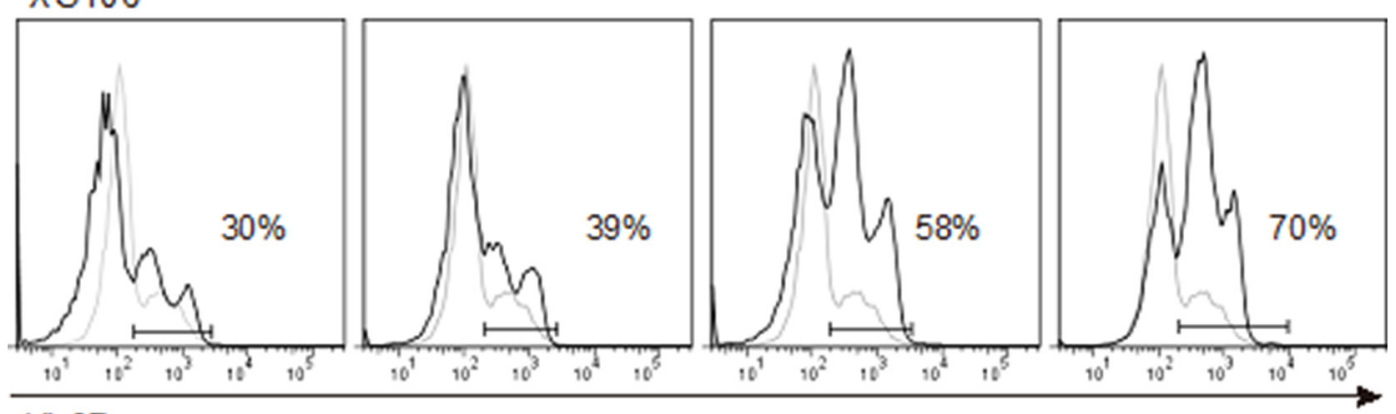

Ki-67

B

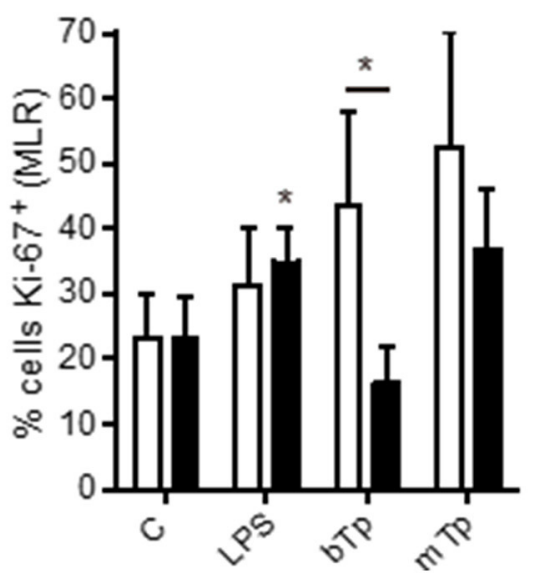

FIGURE 4 | T cell proliferation stimulated by BMDCs or XS106 cells conditioned by different infective stages of T. cruzi. BMDCs or XS106 cells were cultured with medium or different stimuli for $24 \mathrm{~h}$. Then, cells were washed, irradiated and cocultured with LN cells enriched in T-cells at 1:10 APCs:LN cells ratio for 3 days. Cell proliferation was assessed by intracellular detection of Ki-67 in T cells obtained from LNs by FACS. One independent experiment of three is represented by histograms. Gray lines show negative controls for the staining, while black lines correspond to cells stained with specific Ab (A). Cultures were set up in triplicate, and results are expressed as the mean \pm SEM (B). ${ }^{*} p<0.05$; two-way ANOVA and Bonferroni's post-hoc correction.

a mature/activated phenotype. That difference opened the possibility of exploring the effect of the parasite in DCs at different differentiation/activation statuses, showing that the XS106 cell line and BMDCs responded differently to T. cruzi.

mTps are transiently involved in the infection during vectorial transmission. Infection occurs via the skin, and after mTp entry into the host, the parasite suffers intracellular multiplication, as amastigotes and then bTp are present at the site of infection, interacting with resident APCs from the skin or migratory APCs. During this process, the parasite probably conditions the response triggered at the very beginning of the infection at the portal of entry. However, there are no reports yet showing skin APCs as a niche for parasite multiplication. Extended literature describes the wide range of tissues where T. cruzi resides in the host (Melo and Brener, 1978; Silva Pereira et al., 2019). Interestingly, a previous study in humans has demonstrated 
that the parasite can persist in the skin, giving cutaneous lesions associated with the presence of multiplicative amastigotes (Hemmige et al., 2012).

In relation to APCs, it was previously demonstrated in humans that T. cruzi modulates the activation state of MoDCs in vitro (Van Overtvelt et al., 1999) and also during experimental infection (Chaussabel et al., 2003). Our results in the XS106 cell model propose that the basal activation phenotype displayed by mature DCs is not negatively modified by the interaction with the parasite, either mTp nor bTp. Basal IL10 production was particularly reduced by mTp concomitantly with an enhanced antigen presentation activity, suggesting that mature epidermal DCs conserved their functional properties while interacting with the parasite (both bTp and mTp). In BMDCs, no important changes were detected in comparison to control cells, as previously reported for bTp (Poncini et al., 2008 , 2010). However, we found that mTp increased TNF- $\alpha$ production and also antigen presentation in these cells. All results together suggest that $T$. cruzi, depending on the infective stage and the activation/maturation status of the APC, could polarize the immune response during the encounter.

Interestingly, invasion and multiplication of the parasite changed depending on the number of parasites added to cell cultures, the infective stage, and the DCs as recipient cell, demonstrating the importance of the niche the parasite finds to settle up and disseminate during infection. However, Tp invasion and the possibility of the parasite multiplying inside the cell would not be conditioning the antigen-presenting capacity in these two types of DCs, since mTp enhanced the lymphoproliferative capacity of both BMDCs and XS106 cells independently of the percentage of cell infection.

Previous reports with mTp obtained from culture or the vector and bTp from mouse infection showed differences in the immune response associated with the stage of the parasite involved. In a dog experimental model, animals presented enhanced cardiac parasitism and inflammatory response when infected with bTp, suggesting that the inoculum source affects the immunopathological aspects of Chagas disease (de Souza et al., 2014). In addition, it was also demonstrated in a mouse model of infection that bTps were more virulent than culture mTps, via both oral and peritoneal inoculation (Dias et al., 2013), concluding and remarking on the importance of the stage used, its origin, and the model of the infection in order to study the immune response.

Unpublished results from our group demonstrate that the infection with mTp displays reduced cell infiltrate and response at the site of infection (Gutierrez, manuscript under redaction) in comparison with the response described for bTp (Poncini and González-Cappa, 2017). Here, we studied functional properties of BMDCs and XS106 cells stimulated with mTp or bTp in an MLR and found that mTp improved the capacity to induce lymphoproliferation in both cells. In addition, we found that bTp did not impair the antigen-presenting property of XS106 cells, as reported for BMDCs (Poncini et al., 2008), and also that mTps enhance the immunogenicity of BMDCs. These data suggest that infective $T$. cruzi stages could interact and induce diverse effects in different APCs. One interesting conclusion emerging from these results is that apparently, $\mathrm{mTp}$, in contrast to $\mathrm{bTp}$, does not exert tolerogenic signals on DCs. In addition, the tolerogenic properties of bTp seem to be effective only in early differentiated DCs (BMDCs) resembling Mo-DCs in experimental models (Lutz et al., 2016). Those differences between $\mathrm{bTp}$ and $\mathrm{mTp}$ propose that parasite stages associated to the type of transmission could condition the early immune response and, in part, the outcome of the infection. When the vectorial transmission occurs, the first interaction of the innate immune system with the parasite is with $\mathrm{mTp}$, a stage that, according to our results, would not affect APC immunogenicity. In contrast, when bTps are responsible for the infection (blood transfusion or congenital transmission), its interaction with steady-state DCs or Mo-DCs (in general, monocytes reach inflammatory foci, Poncini and González-Cappa, 2017) could probably impair antigen presentation by these cells, and this could make the immunity against the parasite not strong enough to control T. cruzi dissemination.

The importance of the study of $T$. cruzi-APCs interactions opens knowledge to better understand the strategies the parasite has developed in order to evade immunity. In addition, these are key topics on the road to developing new strategies for immunotherapy.

\section{DATA AVAILABILITY STATEMENT}

The datasets generated for this study are available on request to the corresponding author.

\section{ETHICS STATEMENT}

The animal study was reviewed and approved by $\mathrm{CD} \mathrm{N}^{\circ}$ 04/2015 approved by the University of Buenos Aires's Institutional Committee for the Care and Use of Laboratory Animals (CICUAL).

\section{AUTHOR CONTRIBUTIONS}

BG, MR, SG-C, and CP contributed the conception and design of the study. BG and CP performed the experiments and analyzed data. EL conducted the epimastigote cultures. BG and CP wrote sections of the manuscript. All authors contributed to manuscript revision and read and approved the submitted version.

\section{FUNDING}

This work was supported by Consejo Nacional de Investigaciones Científicas y Técnicas (CONICET), Fundación Bunge \& Born, and Universidad de Buenos Aires (UBACyT 2017 20020160100117BA), Argentina.

\section{ACKNOWLEDGMENTS}

We thank Eduardo Gimenez, Ricardo Chung, and, Marianela Lewicki for technical assistance. 


\section{REFERENCES}

Alba Soto, C. D., Mirkin, G. A., Solana, M. E., and González Cappa, S. M. (2003). Trypanosoma cruzi infection modulates in vivo expression of major histocompatibility complex class II molecules on antigen-presenting cells and T-cell stimulatory activity of dendritic cells in a strain-dependent manner. Infect. Immun. 71, 1194-1199. doi: 10.1128/IAI.71.3.1194-11 99.2003

Cestari, I., Ansa Addo, E., Deolindo, P., Inal, J. M., and Ramírez, M. I. (2012). Trypanosoma cruzi immune evasion mediated by host cellderived microvesicles. J. Immunol. 188, 1942-1952. doi: 10.4049/jimmunol.11 02053

Chaussabel, D., Pajak, B., Vercruysse, V., Bisseyé, C., Garzé, V., Habib, M., et al. (2003). Alteration of migration and maturation of dendritic cells and T-cell depletion in the course of experimental Trypanosoma cruzi infection. Lab. Invest. 83, 1373-1382. doi: 10.1097/01.LAB.0000087587.93781.6F

da Costa, T. A., Silva, M. V., Mendes, M. T., Carvalho-Costa, T. M., Batista, L. R., Lages-Silva, E., et al. (2014). Immunomodulation by Trypanosoma cruzi: toward understanding the association of dendritic cells with infecting TcI and TcII populations. J. Immunol. Res. 2014:962047. doi: 10.1155/2014/ 962047

de Souza, S. M., Vieira, P. M., Roatt, B. M., Reis, L. E., da Silva Fonseca, K., Nogueira, N. C., et al. (2014). Dogs infected with the blood trypomastigote form of Trypanosoma cruzi display an increase expression of cytokines and chemokines plus an intense cardiac parasitism during acute infection. Mol. Immunol. 58, 92-97. doi: 10.1016/j.molimm.2013.11.007

Dias, G. B., Gruendling, A. P., Araújo, S. M., Gomes, M. L., and Toledo, M. J. (2013). Evolution of infection in mice inoculated by the oral route with different developmental forms of Trypanosoma cruzi I and II. Exp. Parasitol. 35, 511-517. doi: 10.1016/j.exppara.2013.08.013

Gonzalez Cappa, S. M., Bijovsky, A. T., Freilij, H., Muller, L. A., and Katzin, A. M. (1981). Aislamiento de una cepa de Trypanosoma cruzi a predominio de formas delgadas en la Argentina. Medicina 41, 119-120.

Hemmige, V., Tanowitz, H., and Sethi, A. (2012). Trypanosoma cruzi infection: a review with emphasis on cutaneous manifestations. Int. J. Dermatol. 51, 501-508. doi: 10.1111/j.1365-4632.2011.05380.x

Isola, E. L. D., Lammel, E. M., and González Cappa, S. M. (1986). Trypanosoma cruzi: differentiation after interaction of epimastigotes and Triatoma infestans intestinal homogenate. Exp. Parasitol. 62, 329-335. doi: 10.1016/0014-4894(86)90039-1

Lutz, M. B., Inaba, K., Schuler, G., and Romani, N. (2016). Still alive and kicking: in-vitro-generated GM-CSF dendritic cells! Immunity 19, 1-2. doi: 10.1016/j.immuni.2015.12.013

Melo, R. C., and Brener, Z. (1978). Tissue tropism of different Trypanosoma cruzi strains. J. Parasitol. 64, 475-482. doi: 10.2307/3279787

Merad, M., Sathe, P., Helft, J., Miller, J., and Mortha, A. (2013). The dendritic cell lineage: Ontogeny and function of dendritic cells and their subsets in the steady state and the inflamed setting. Annu. Rev. Immunol. 31, 563-604. doi: 10.1146/annurev-immunol-020711-074950
Mohan, J., Hopkins, J., and Mabbott, N. A. (2005). Skin-derived dendritic cells acquire and degrade the scrapie agent following in vitro exposure. Inmunology 116, 122-133. doi: 10.1111/j.1365-2567.2005.02207.x

OMS (2019). Organización Mundial de la Salud. La Enfermedad de Chagas (Tripanosomiasis Americana). Available online at: http://www.who.int/ mediacentre/factsheets/fs340/es/

Otsuka, M., Egawa, G., and Kabashima, K. (2018). Uncovering the mysteries of langerhans, cells, inflammatory dendritic epidermal, cells, and monocytederived langerhans cell-like cells in the epidermis. Front. Immunol. 30:1768. doi: 10.3389/fimmu.2018.01768

Planelles, L., Thomas, M. C., Marañón, C., Morell, M., and López, M. C. (2003). Differential CD86 and CD40 co-stimulatory molecules and cytokine expression pattern induced by Trypanosoma cruzi in APCs from resistant or susceptible mice. Clin. Exp. Immunol. 131, 41-47. doi: 10.1046/j.1365-2249.2003.02022.x

Poncini, C. V., Alba Soto, C., Batalla, E., Solana, M. E., and González Cappa, S. M. (2008). Trypanosoma cruzi induces regulatory dendritic cells in vitro. Infect. Immun. 76, 2633-2641. doi: 10.1128/IAI.01298-07

Poncini, C. V., Giménez, G., Pontillo, C. A., Alba-Soto, C. D., de Isola, E. L., Piazzón, I., et al. (2010). Central role of extracellular signalregulated kinase and Toll-like receptor 4 in IL-10 production in regulatory dendritic cells induced by Trypanosoma cruzi. Mol. Immunol. 47, 1981-1988. doi: 10.1016/j.molimm.2010.04.016

Poncini, C. V., and González-Cappa, S. M. (2017). Dual role of monocyte-derived dendritic cell in Trypanosoma cruzi infection. Eur. J. Immunol. 47, 1936-1948. doi: 10.1002/eji.201646830

Poncini, C. V., Illarregui, J. M., Batalla, E. I., Engels, S., Cerliani, J. P., Cucher, M. A. van Kooyk, Y., et al. (2015). Trypanosoma cruzi infection imparts a regulatory program in dendritic cells and T cells via galectin-1-dependent mechanisms. J. Immunol. 195, 3311-3324. doi: 10.4049/jimmunol.1403019

Silva Pereira, S., Trindade, S., De Niz, M., and Figueiredo, L. M., (2019). Tissue tropism in parasitic diseases. Open Biol. 31:190036.doi: 10.1098/rsob. 190124

Van Overtvelt, L., Vanderheyde, N., Verhasselt, V., Ismaili, J., De Vos, L., Goldman, M., et al. (1999). Trypanosoma cruzi infects human dendritic cells and prevents their maturation: inhibition of cytokines, HLA-DR, and costimulatory molecules. Infect. Immun. 67, 4033-4040. doi: 10.1128/IAI.67.8.4033-4040.1999

Conflict of Interest: The authors declare that the research was conducted in the absence of any commercial or financial relationships that could be construed as a potential conflict of interest.

Copyright (C) 2020 Gutierrez, Lammel, Ramirez, González-Cappa and Poncini. This is an open-access article distributed under the terms of the Creative Commons Attribution License (CC BY). The use, distribution or reproduction in other forums is permitted, provided the original author(s) and the copyright owner(s) are credited and that the original publication in this journal is cited, in accordance with accepted academic practice. No use, distribution or reproduction is permitted which does not comply with these terms. 